\title{
A propos de la littérature francophone/ des littératures francophones: quelques aspects de la question
}

\section{Laurence Malingret}

\section{(2) OpenEdition}

Journals

\section{Édition électronique}

URL : http://journals.openedition.org/studifrancesi/27073

DOI : 10.4000/studifrancesi.27073

ISSN : 2421-5856

Éditeur

Rosenberg \& Sellier

\section{Édition imprimée}

Date de publication : 31 décembre 2006

Pagination : $542-547$

ISSN : 0039-2944

\section{Référence électronique}

Laurence Malingret, «A propos de la littérature francophone/ des littératures francophones: quelques aspects de la question », Studi Francesi [En ligne], 150 (L | III) | 2006, mis en ligne le 30 novembre 2015, consulté le 08 novembre 2020. URL : http://journals.openedition.org/studifrancesi/27073 ; DOI : https://doi.org/10.4000/studifrancesi.27073

\section{(c)}

Studi Francesi è distribuita con Licenza Creative Commons Attribuzione - Non commerciale - Non opere derivate 4.0 Internazionale. 


\section{A propos de la littérature francophonel des littératures francophones: quelques aspects de la question}

On observe actuellement dans le milieu universitaire un intérêt croissant pour les littératures francophones et pour leur enseignement. C'est à notre avis d'autant plus positif que l'enseignement de ces littératures oblige à repenser certains présupposés théoriques et méthodologiques. Nous pensons que les théories systémiques représentent une possibilité parmi d'autres, mais particulièrement éclairante, pour aborder celles-ci. Nous pensons à l'approche sociologique de la littérature de Jacques Dubois ou de Pierre Bourdieu et à la théorie des polysystèmes de l'école de Tel Aviv principalement qui, s'éloignant d'une conception idéaliste et atemporelle de la littérature et du textocentrisme, considèrent la littérature comme un système socioculturel et comme un acte de communication dont la définition est fonctionnelle et déterminée par les relations entre les différents facteurs du système.

\section{Une question de terminologie}

L'étude des littératures francophones pose plusieurs questions à commencer par la question de la terminologie. Il semble que le terme francophone se soit imposé pour désigner les littératures en langue française produites hors de France. Mais les exceptions ne manquent pas: l'anthologie de Jean-Louis Joubert, Littérature francophone, englobe les productions de l'Hexagone et l'ouvrage de Christian Berg et Pierre Halen s'intitule Littératures belges de langue française. Ces deux exemples sont assez représentatifs d'une situation en mouvance et de deux idéologies. D'une part, la perception d'une littérature unique autour d'un critère linguistique, et de l'autre, une pluralité tendant à l'autonomie. Le concept même de francophonie est assez fluctuant. Le terme recouvre parfois des réalités différentes selon l'accent tantôt mis sur l'aspect politique, tantôt sur l'aspect culturel, tantôt sur l'aspect économique. C'est un terme descriptif mais il est aussi employé dans un sens plus militant, comme le souligne le Petit Robert en lui attribuant la nuance «en faveur de la langue française». Le combat de la France, sous la banderole de la francophonie, pour la défense de la langue française, n'est pas gratuit, ni d'ailleurs la collaboration des pays francophones qui cache des enjeux d'ordre divers.

\section{Littérature francophone et littérature nationale}

La francophonie littéraire est donc un ensemble recouvrant des réalités culturelles diverses souvent perçu dans un rapport de centre-périphérie qui, théoriquement, inclut bien sûr la production française, mais qui, dans la pratique, semble souvent être utilisé comme un raccourci pour parler des littératures de langue française hors de France.

L'approche théorique, descriptive, analytique des littératures francophones est assez récent, et le statut, loin d'être consolidé, de ces études dans l'institution universitaire en est le reflet. Il est en effet frappant que les études spécifiques de littératures francophones ont rencontré et rencontrent encore dans l'actualité d'énormes difficultés à s'imposer en tant que discipline spécifique, non seulement en France, mais 
également dans les propres pays francophones comme la Belgique où, il n'y a pas si longtemps, un étudiant en lettres modernes pouvait se licencier en ignorant tout de la production belge confinée dans les cours à option. Cette résistance peut être expliquée de différentes façons, à commencer par des raisons historiques, et concrétise la complexité de la question. Envisager les littératures périphériques oblige aussi à repenser des concepts pratiques pour l'enseignement traditionnel des littératures telles que littérature nationale ou la pertinence du critère et du découpage linguistiques. Prenons l'exemple de la littérature africaine en français. Est-il plus performant d'envisager cette production comme appartenant à une littérature plurilingue (langues vernaculaires, anglais), à un ensemble de littératures nationales ou à la francophonie littéraire? C'est aussi redéfinir le canon en acceptant un canon hybride né d'apports extérieurs, un modèle de culture métissée. D'autre part, la définition des limites et le statut d'une littérature sont en bonne partie le résultat d'une polémique idéologique, le concept de littérature nationale n'y échappe pas. Cette construction, redevable en partie d'une perception romantique de la réalité, est pratique et inévitable, car elle forme partie de nos repères et sous-tend toute réflexion comparatiste envisageant les relations littéraires «inter-nationales», mais doit être utilisée avec précaution.

C'est en effet un concept qui a surgi dans des circonstances précises et qui ne peut être appliqué sans nuance à l'infinie variété de cas de figure littéraire dans le temps et dans l'espace. Ainsi, il est évident que parler de littérature nationale a posteriori peut être remis en question. Peut-on parler de littérature nationale française au moyen-âge? Et que représente ce concept actuellement, en rapport avec l'évolution de la nation en général (et le rejet qu'il peut susciter face à une perception globalisante du monde), sachant que toutes les nations n'ont pas une littérature nationale ni une langue nationale et que toutes les littératures ne correspondent pas à une nation?

Considérer, comme c'est parfois le cas, que la littérature nationale est celle produite dans la langue de la nation, est valable mais à nuancer, notamment dans la perspective francophone. Le critère linguistique est sans conteste un critère pertinent pour la définition des littératures nationales, notamment pour les littératures qui se sont consolidées en tant que telles au XIXème siècle, dans la mesure où la langue forme une partie importante de l'identité culturelle, mais parler de littérature nationale implique d'autres composantes comme la reconnaissance d'une perception commune et croisée du «national» et du «littéraire». De nombreuses questions se posent: Existent-ils des systèmes littéraires nationaux où la langue originaire de la communauté n'est pas la même que la langue littéraire? Existe-t-il une littérature sans ancrage national à proprement parler? Quels rapports existent-ils entre les frontières politiques et les frontières littéraires? Comment définir la littérature nationale congolaise sachant que la plupart des questions concernant la délimitation et la caractérisation des littératures africaines en français incluent le rapport ambigu des systèmes dominants et des systèmes émergents qui incorporent malgré tout des éléments conceptuels du système combattu et colonisateur?

A.-M. Thiesse a mis en évidence le rôle de la littérature dans la formation des nations et il est utile de se pencher sur les rapports fluctuants de dépendance entre littérature et organisation sociale et politique. La délimitation des littératures nationales est aussi un exercice de construction historique liée aux intêrets de la mémoire collective et de l'identité nationale. C'est dans ce cadre que peuvent etre envisagées les littératures émergentes ou consolidées dans la sphère francophone, ainsi que les relations internationales entre centre et périphérie et les transferts culturels d'éléments métamorphosés selon, notamment, l'état de construction de la littérature nationale et l'autonomie du champ littéraire. L'introduction d'éléments étrangers peut ainsi être ressenti comme une menace pour l'intégrité du système ou comme un enrichissement culturel qui recouvre néanmoins l'impérialisme du système. 
On peut considérer que le concept de littérature nationale au sens strict du terme est le reflet d'une perception eurocentriste et romantique de la littérature basée sur le lien entre littérature, langue et frontières géopolitiques et, de ce fait, n'est pas l'outil le plus performant pour rendre compte d'une réalité autre que l'occidentale voire même pour des situations européennes minoritaires. Naftoli Bassel (1991) considère même que les littératures nationales sont liées à une époque précise, celle de la formation du capitalisme et de la cristallisation d'un groupe ethnique en nation et que la littérature suisse, canadienne ou belge en français sont des littératures ethniques. Sans aucun doute les approches systémiques, en incluant le principe d'hétérogénéité, évitent de considérer ces situations comme des exceptions ou des cas particuliers. Peut-être serait-il dès lors plus efficace de parler, pour la Belgique par exemple, non d'une littérature nationale, mais concrètement des littératures en Belgique. Parler des littératures en Belgique n'évacue pas le problème, mais le déplace et permet de situer la question primordiale de la littérature nationale dans une approche plus strictement historique.

\section{Question de typologie}

Les difficultés réelles que posent les littératures francophones sont proportionnelles à leur intérêt théorique pour l'étude du fonctionnement des systèmes littéraires. En effet, nous pensons ici aux littératures des ex colonies, elles représentent une belle occasion d'analyser le phénomène des littératures émergentes, leur confrontation avec le système dominant, qui peut les exclure du champ de la légitimité ou les marginaliser, et leur cheminement vers l'autonomie (les littératures africaines) ou vers la disparition (au Proche Orient). Il faut pour cela prendre conscience de la complexité et de la variété des cas de figure et dépasser les deux tendances dominantes dans l'approche de la francophonie: d'une part le discours «idéalisant», qui perçoit la production hors de France comme un effet du rayonnement culturel français avec en retour un effet d'enrichissement du patrimoine, et le discours de la mauvaise conscience post coloniale qui oppose et impose les langues (rejet du français au profit des langues vernaculaires). L'accent est ainsi mis soit sur l'unité de la francophonie littéraire ou sur le rejet de son rôle. Pour notre part, nous croyons que les modèles théoriques du polysystème et du champ littéraire sont particulièrement performants pour ce genre de configuration (modèle culturel hybride né d'une situation de colonisation) et que l'étude des littératures francophones peut se bénéficier d'une approche comparatiste. Il s'agit d'observer les facteurs endogènes, comme le nationalisme, et les facteurs exogènes, comme la francophonie, conséquence de la colonisation et d'envisager le phénomène du transfert dans toutes ses dimensions (réception, réinterprétation, pragmatisation, fictionalisation, métamorphose de biens culturels). En fait, l'étude des littératures francophones est bel et bien un exercice de littérature comparée dont la spécificité est l'unité (relative) de langue.

Une caractéristique importante de l'étude des littératures francophones est sa complexité croissante, il ne s'agit donc pas de vouloir l'embrasser dans un exercice de littérature comparée global, mais de définir une typologie exhaustive qui permette de prendre en compte les différentes variantes. En observant les publications les plus récentes, on observe que de nombreux critères sont possibles et sont tous plus ou moins performants, du critère religieux (le monde musulman d'expression française) au critère purement linguistique, d'une grande complexité et qui doit tenir compte des politiques linguistiques. Les critères géo-politiques (filiation/éloignement) et linguistiques sont néanmoins les plus utilisés. En général on distingue ainsi quatre cas de figure: les littératures de souche francophone (Belgique, Suisse, Canada), les dicho- 
tomies linguistiques avec une langue apparentée (les créoles) ou non (arabe, langue vernaculaire africaine) et le rayonnement culturel ponctuel (au Liban par exemple). Il est intéressant de s'interroger sur la formation des univers symboliques et de leur relation avec la littérature.

\section{Univers symbolique et champ littéraire}

Il peut exister un univers symbolique homogène (les situations de créolisation) ou plusieurs univers symboliques (les situations de colonisation) s'exprimant par le biais de littératures en langues différentes (Cf. à ce propos BEnIAmino, 1999). Il faut par ailleurs tenir compte de certaines évolutions à ce niveau. Prenons le cas de la littérature belge qui s'est forgé grâce aux écrivains flamands un univers symbolique particulier (les brumes du Nord, le mysticisme, etc.), qui est d'ailleurs à la base de la reconnaissance de la spécificité belge vu de l'extérieur et de l'Hexagone en particulier. Or, la situation politique a métamorphosé un découpage linguistique de type social (l'élite parlant français, le peuple parlant flamand dans le Nord et wallon dans le Sud) en un découpage de type géographique et politique. Dès lors l'univers symbolique flamand n'étant plus exprimé par la voie francophone, les écrivains francophones ont eu toutes les peines du monde à se redéfinir d'où, en partie, les assimilations d'écrivains au système littéraire français (gommage de particularités linguistiques et surtout coupure du terreau social belge) d'une part, et la définition par le manque ou par la négation que représente la belgitude, d'autre part. D'autre part les écrivains flamands contemporains qui ont choisi d'écrire en français, peu nombreux, ont développé un rapport à la langue complexe qui parfois est devenu le moteur de leur oeuvre. Un exemple particulièrement frappant est celui du dramaturge Paul Willems qui, de sa position périphérique, a eu toutes les peines du monde à être reconnu dans le champ littéraire francophone, selon la terminologie de Pierre Bourdieu. Son oeuvre a en effet longtemps été boudée par le public français mais reconnue par le biais de ses traductions en langues germaniques. Paul Willems poursuit la tradition du théâtre onirique belge incarné par le symboliste Maurice Maeterlinck. Son monde particulier et séduisant se situe aux lisières du rêve et de la réalité. Univers aux frontières floues, il est peut-être le refuge qui sublime le malaise d'une identité difficile à revendiquer. Flamand et francophone, Paul Willems est le représentant, comme il le souligne lui-même, «d'une race en voie de disparition» (in P. Emond, H. Ronse, F. van de Kerckhove, 1984: 230), tiraillée et malmenée par une société en pleine mutation. Sa formation partagée entre deux langues, son statut incommode d'auteur flamand écrivant en français, ont créé en lui un déchirement linguistique, immanquablement marqué dans son écriture prodigieusement inventive. C'est un lieu commun de dire que le langage est au cœur des préoccupations d'un écrivain, et pourtant chez Paul Willems, il est véritablement le protagoniste et constitue une des clés les plus évidentes de son oeuvre. Quoi d'étonnant venant d'un écrivain qui, à 19 ans, débarquant à Paris, s'inquiète «Je ne connais pas le français, je connais mal le flamand, je n'ai pas de langue». (op. cit., 1984: 229). Paul Willems s'est exprimé sur son rapport particulier à la langue, sur la possibilité de ressentir avant d'exprimer, sur son rêve d'une langue idéale qui ne structurerait pas sa perception de la réalité, mais aussi sur sa peur des mots sclérosés, du «voyage inutile» de l'écriture qu'il a néanmoins entrepris et expliqué.

Ces deux tendances aux limites de la perfection et de l'échec s'entrecroisent dans une écriture souvent qualifiée de poétique. Son rejet du vocabulaire abstrait pour privilégier la dimension concrète et sonore de la langue et sa volonté de redonner vie aux mots usés et aux expressions figées, recourant à la création en toute liberté si besoin 
s'en fait sentir, n'y sont pas étrangers. Corollairement à ce travail poétique que la simple énumération des titres ou des noms des personnages concrétise, l'œuvre de Paul Willems se penche sur l'efficacité communicative du langage. Force est de constater que de ce point de vue, ses pièces sont de plus en plus pessimistes et débouchent sur une véritable impasse. Les nombreux dysfonctionnements de la communication, perceptibles dès ses premières pièces, des comédies féeriques légèrement amères, aboutissent à la personnalisation et la fragmentation du langage élevées au plus haut niveau dans Les Miroirs d'Ostende et dans La Ville à voile, textes plus achevés et plus sombres aussi, où même la poésie est remise en question.

Son théâtre porte ainsi les stigmates d'une langue aliénante et impuissante qui isole plus qu'elle n'unit, de même que la nostalgie (et l'espoir) d'une langue riche et poétique qui dit au-delà du sens conventionnel des mots, d'un langage à la fois désespérant et irrésistible, en somme, profondément humain.

\section{Problématique des littératures francophones}

Les littératures francophones représentent des situations littérairement et culturellement très différentes, mais certaines constantes et problématiques peuvent être observées pour un certain nombre d'entre elles. Bien évidemment certaines questions peuvent être posées pour toute situation littéraire:

- La «décolonisation» littéraire (de la littérature coloniale à la rupture et autonomie dans les normes et dans les infrastructures notamment de l'édition et de l'enseignement).

- Le mouvement de balancier entre phase centripète (tendant à l'assimilation avec le champ littéraire français) et centrifuge (tendant à l'autonomie), selon la terminologie de L. Grigorievitch Andreev.

- Le statut particulier de la langue française (langue de l' écrit, langue de combat, langue de prestige, langue officielle), le statut particulier de la production littéraire en français (en position d'opposition avec la littérature orale ou écrite en langue vernaculaire, production de prestige, de combat,...) et variations du rapport entre production littéraire et contexte social (rôle des élites). La possible diglossie dans les ex-colonies. L'insécurité linguistique qui génère des modèles antagonistes: le purisme (visant à l'assimilation) ou le «baroque» (visant à la transgression, à la rupture).

- Dynamique de la production littéraire (réel moteur culturel et politique comme lors de la Révolution Tranquille au Québec ou production intermittente comme au Proche Orient) et positionnement des politiques culturelles particulières (rôle des organismes et institutions officielles ).

- Système de légitimation des oeuvres. La question de la norme et sa répercussion différenciée dans la production et dans la réception. Infrastructure locale et générale de reconnaissance et de diffusion (institutions, presse, enseignement, etc.). Difficulté et importance de la reconnaissance fondée sur des normes spécifiques de la valeur littéraire par l'institution française.

- Particularité de la réception et des fonctions fluctuantes d'reuvres hybrides se situant entre les normes littéraires dominantes du système français et des normes propres. La question du public cible (le lecteur idéal et le lecteur réel) et les conditions de diffusion et de lecture des oeuvres. Distance entre l'espace d'écriture et l'espace de lecture.

- Rôle de la littérature dans la constitution d'un univers symbolique dans ses rapports à la société et dans ses rapports à la francophonie.

- La dimension transnationale de la littérature. La question de l'écriture de l'exil. L'apparition d'une nouvelle carte mondiale de la littérature. 
- Les rapports centre-périphérie et périphérie-périphérie (l'exemple des intenses contacts culturels au sens large entre le Québec et la Wallonie par exemple).

Littérature(s) francophone(s) au singulier ou au pluriel, la francophonie littéraire est le lieu de l'unité et de la diversité, et nous retrouvons dans la critique toutes les options possibles: de la négation d'une francophonie, qui n'a en commun que ses colloques, à la cohérence d'une littérature à la fois une et plurielle. Entre les deux, se situe la thèse comparatiste, même s'il est vrai que la spécialisation que l'on observe dans la recherche (à travers les publications qui délimitent de façon de plus en plus précise le terrain de l'analyse et prennent en compte des facteurs culturels spécifiques - la littérature francophone africaine par exemple semble s'associer aux études d'africanisme) semble reléguer au passé des entreprises comparatistes globales telles que celle d'A. Viatte. La démarche de Riesz et Ricard (1991) qui envisagent le champ littéraire togolais comme un ensemble cohérent et multilingue ouvre également de nouvelles perspectives dans l'approche de la réalité post coloniale. Nous pensons en tout cas que la francophonie littéraire représente un formidable terrain pour l'étude des transferts culturels et des relations asymétriques entre systèmes littéraires et que la multiplication des publications la concernant n'est certainement pas le fruit du hasard.

LAURENCE MALINGRET

\section{Références bibliographiques}

W. BAL, Unité et diversité de la langue français, in A. Reboullet, et M. Tétu, Guide culturel - I, Civilisations et littératures d'expression française, Québec, Hachette Presses de l'Université de Laval, 1977.

N. Bassel, National Literature and Interliterary System, «Poetics Today», 1991, 12 (4), pp. 773-780.

M. Beniamino et D. DeRobillard (éds), Le français dans l'espace francophone, Paris, Champion, 1993.

M. Beniamino, La francophonie littéraire. Essai pour une théorie, Paris, L'Harmattan, 1999.

Chr. Berg, P. HaLen (Dir), Littératures belges de langue française (1830-2000), Bruxelles, Le Cri édition, 2000.

P. BouRdieu, Les Règles de l'art. Genèse et structure du champ littéraire, Paris, Le Seuil, 1992.

J. DuboIs, L'Institution de la literature; Introduction à une sociologie, Paris, Nathan, 1978.

P. Edmond, H. Ronse, F. Van De Kerckhove (éds), Le monde de Paul Willems, Bruxelles, Labor, 1984.

L. Even-Zohar, Polysistem Studies, «Poetics Today», 1990, 11, nº 1.

P. Halen, M. Otten (éds), Lectures de Paul Willems, in «Textyles», 1988, n 5, Bruxelles.

J.-L. JOUBERT, Littérature francophone, Paris, Nathan, 1992.

A. Reboullet et M. Tetu, Guide culturel. Civilisations et littératures d'expression française, Québec Hachette, PU Laval, 1977.

J. RIESZ, A. RicARD, Le champ littéraire togolais, Bayreuth, E. Breilinger, 1991.

R. SHefFy, The concept of canonicity in Polysystem Theory, «Poetics Today», 1990, 11 (3), pp. $511-52$.

A.-M. Thiesse, La création des identités nationales», Paris, Seuil, 1999.

A. Viatte, La Francophonie, Paris, Larousse, 1969. 